\title{
On the Heuristics of the Higgs Mechanism
}

\author{
Sébastien Rivat* \\ The online publication is available at Springer via \\ http://link.springer.com/article/10.1007\%2Fs10838-014-9258-4 \\ DOI: $10.1007 / \mathrm{s} 10838-014-9258-4$

\begin{abstract}
This article has two aims. First, I undertake an extensive review of the Higgs mechanism and its connections with spontaneous symmetry breaking and the Goldstone theorem. I take the opportunity to expound and discuss a certain number of philosophical issues, amongst them surplus structure and redundancies. Second, I offer a defence of the metaphor according to which 'gauge fields eat Goldstone bosons to gain a mass' as sensible rather than merely misleading. It is sensible because there is a direct physical correspondence between the longitudinal polarization of massive gauge fields and Goldstone bosons, which is not merely set by a gauge-fixing procedure. In these terms, I wish to argue that the mechanism which allows for the discovery of the Higgs boson has more than merely heuristic and methodological virtue.
\end{abstract}

Keywords: Higgs mechanism, Goldstone bosons, Spontaneous symmetry breaking, Gauge theories, Quantum field theories, Realism

\section{Introduction}

The Higgs mechanism is famous for explaining with mathematical elegance why fields are given mass in the quantum field theories (QFTs) of particle physics. At its roots lies the less well-known mechanism of spontaneous symmetry breaking (SSB). Its principle is simple: the theoretical laws governing physical phenomena admit symmetries that are not empirically manifested in the physical states. SSB was first applied to high energy physics through the Goldstone Theorem (Goldstone, 1961). This theorem predicts the appearance of massless, spinless particles, the so-called Goldstone bosons, when global symmetries are

\footnotetext{
*Department of Philosophy, Columbia University, 10027 New York, USA. Email: sr3109@columbia.edu
} 
broken. Historically, no such particles were detected in the particle spectrum of high energies, which led to serious doubts about the role of SSB. Given this impasse, the Higgs mechanism kills two birds with one stone: (1) it solves the issue of experimental detection by assuming that all the Goldstone bosons are "eaten up" by some gauge fields; and (2) it allows the existence of massive gauge fields, while keeping the theory gauge invariant which is necessary for a suitable theory of interactions between fields.

The difficulty with the Higgs mechanism lies in being interpreted realistically. (1) My first concern is that the whole mechanism appears rather "ad hoc": one assumes the existence of supplementary scalar fields and a suitable pattern of SSB in order to give the appropriate masses to the gauge fields. The recent detection of the Higgs boson reduces this worry, though some of the bosons' characteristics remain unknown. (2) Secondly, the issue of gauge symmetries is also of concern. These are usually referred to as the transformations between physically equivalent states of affairs and therefore redundant descriptions. It thus appears artificial to focus the Higgs mechanism on breaking these symmetries. (3) My third concern relates to the "just-so stories" (Earman, 2004b, 1239) told about the mechanism, including the metaphorical idea that some gauge bosons become massive by "eating" the Goldstone bosons. These three concerns have led philosophers to acknowledge the methodological and heuristic value of the Higgs mechanism, although severe doubt has been cast on the possibility of finding a suitable realistic interpretation of it (e.g., Morrison, 2003; Lyre, 2008).

Of course, the term 'realistic' remains highly controversial, and I do not intend to add to this debate. One might simply keep in mind that some entities and structures are believed to exist and manifest themselves independently from the description. I wish to focus on a weak meaning of "independence"; namely, the absence of theoretical ambiguity. This ambiguity may take various forms. (1) The theoretical description is redundant and so we have the choice to decide arbitrarily ("by hand") between equivalent descriptions. This choice, therefore, raises the issue of whether the description is itself arbitrary. (2) Another form of ambiguity is when the theory contains crucial mathematical artefacts or "surplus structure" (Redhead, 2003). In this case, the mathematical framework is larger than required, and the surplus not properly redundant. The ambiguity arises when the surplus structure crucially shapes the theoretical description of physical phenomena, so that one may be unable to assess whether the physics is independent from this surplus. The problem for us is that the Higgs mechanism vividly illustrates both these two philosophical issues, redundancy and surplus structure, whether SSB (Sec. 2), the Goldstone bosons (Sec. 3), or the gauge freedom (Sec. 5.1) are concerned. One may thus be led to form the extreme conclusion that the recent detection of a Higgs-like particle at the Large Hadron Collider experiments (Collaboration, 2012) does not support the physical existence of the mechanism whereby the Higgs bosons arise.

My aim here is not to defend realism at all costs, however, and I will not assume realism. 
I rather aim to tackle the following, more specific question: in what physical sense can the gauge fields be said to absorb the Goldstone bosons? I suspect that many aspects of this question have been overlooked by the catchphrase that the gauge fields "eat descriptive fluff" (Earman, 2004b, 1239), and I will argue that there is an ambiguous physical relation between the gauge fields and the Goldstone bosons which cannot be dismissed easily. In Section 2, I first briefly clarify the definition of SSB, and explain why I will rely on the conventional formulations that are most widely adopted by physicists. The question of the physicality of Goldstone bosons is discussed in Section 3, following a review of the Goldstone theorem. Section 4 reviews the Higgs mechanism and lays the ground for the gauge issues and the Goldstone bosons being "eaten". Section 5 sketches the principal gauge-theoretic issues, and contrasts these with the fact that the Higgs mechanism is currently the best way to describe massive gauge fields. Finally, Section 6 discusses how Goldstone bosons can be said to give mass to gauge fields.

\section{Spontaneous symmetry breaking}

For conceptual clarity, let us first distinguish between at least three ways of understanding SSB in particle physics:

(i) A general 'equation vs. solution' meaning: The general equations which describe a system are invariant under a symmetry whereas typically, an individual solution is not. The free Schrödinger equation is, for instance, invariant under parity, a symmetry not respected by the solution of a single plane wave moving forwards in time.

(ii) A 'lowest energy state' meaning: The system possesses a degenerate lowest energy state; namely, in QFT, many distinct vacua where each vacuum does not possess the symmetry that governs the system.

(iii) A 'phase transition' meaning: A system becomes unstable at a given temperature and experiences a dynamical phase transition. When water freezes, for instance, a phase transition occurs from the liquid to the solid state.

This list is not exhaustive. Nor are the three definitions sharply separated: for instance,

(ii) is a special case of (i). I make this distinction with the underlying idea of laying aside (iii) and focusing mainly on (ii).

Before explaining this point, both (i) and (ii) make salient the fact that SSB implies a sort of gap between the description given by the laws and that given by the solutions. From the perspective of each solution, the symmetry seems to be lost or "hidden". For instance, in case (ii), it cannot be seen by an observer who stands in one particular vacuum (Coleman, 1985). In other words, the law describes symmetrically related possibilities, 
which are disconnected in the world. The symmetry or the solutions may therefore be considered a surplus structure of the theory (or be considered as redundancies when the solutions are equivalent).

In case (ii), which interests us, the existence of many distinct vacua is often dismissed since a QFT is built upon only one vacuum state. One can build a theory on each distinct vacuum, and interpret them as many separate worlds like different homogeneous spin orientations in a ferromagnet (see (2) below). However, there is no observable to distinguish between the different vacua in QFT (more about this in Sec. 3). The many QFT descriptions are thus redundant or, at least, also left physically indistinguishable. So, with ontological parsimony - and this is the physicists' practice - the choice is to pick only one particular vacuum state, with all the arbitrariness that this choice implies.

Perhaps (iii) may come to our rescue here: the initial perturbations that provoke a phase transition ought to determine a preferred vacuum state. This is how the Higgs mechanism is usually interpreted on a cosmological scale. At the origins, initial scalar fields, already coupled to matter, evolve upon their single vacuum. The Universe cools gradually, and modifies smoothly the shape of the scalar potential until a certain age when the phase transition suddenly occurs. The symmetry spontaneously breaks down, the unstable scalar fields "roll" down towards a particular vacuum state like a ball rolling down from the peak of a Mexican hat, and matter gains mass. However, the scenario of a SSB phase transition during the cooling of the Universe both: (1) leads to philosophical issues; and (2) hitherto rests upon speculative theoretical grounds.

(1) The cosmological interpretation of the Higgs mechanism excessively depends on the idea that, to be realistic, the Higgs mechanism needs to be historical, in the sense of ordering and distributing events on a temporal scale. The chronological aspect first: however intuitive, the previous story really copies the logical steps of the modern formulation of the Higgs mechanism (see Sec. 4). The issue is not that the story produces an intuitive visualization, but rather that the chronology makes it necessary to interpret realistically the formal derivation, for instance a Lagrangian description, in any of its parts. The chronology also requires a linear reading that is not necessarily fixed by the formalism. Lastly, the chronology only succeeds if the whole mechanism is interpreted: any discontinuity amounts to losing the causal explanation within the series of events. Yet, numerous issues arise in the derivation of the Higgs mechanism, amongst them gauge freedom (Sec. $5.1)$.

(2) Now let us discuss the grounds for the distribution of events in the Higgs mechanism. I submit that the time-location and the narrative of the events in the Higgs mechanism wrongly gains credits from the analogy that has been made with the spontaneous magnetization of a ferromagnet. In the analogue, when the temperature cools, the many atomic spins in the ferromagnet spontaneously take a specific orientation, and break the initial rotational symmetry given by the random orientations. This analogy 
has been deemed inadequate by numerous philosophers (e.g., Kosso, 2000; Lyre, 2008). One of the strongest reasons against it is that there is no temperature parameter in the Higgs mechanism. Since the cosmological time evolution is based on the cooling of the temperature, so the Higgs mechanism fails to be properly historical.

The two previous arguments do not mean that a historical interpretation is necessarily misleading. These arguments merely clarify that, in the present state of affairs, the cosmological interpretation is speculative and so fails to support a realistic interpretation of the Higgs mechanism. I will accordingly lay aside case (iii). ${ }^{1}$ Stories will, in general, appear to be more convincing tools later (Sec. 6).

Now that the general background of SSB has been established, the ensuing developments will be drawn from a heuristic (or conventional) formulation of QFTs as opposed to the algebraic formulation (AQFT). ${ }^{2}$ The former includes the traditional formalism, the methods and tools of QFTs used by physicists (e.g., see Weinberg, 1996), while the latter - as particularly emphasized by philosophers of physics - formulates and resolves several issues about SSB in terms of abstract algebras of operators: for instance, the consistency of a degenerate vacuum with Wigner's theorem (Earman, 2003, 341; Baker and Halvorson, 2013, 7-9), and with the requirement of a unique vacuum in QFTs (Earman, 2003, 341). The choice of the heuristic formulation is driven by the need to understand better the dynamics of the fields, their interaction, and the issue of gauge symmetry.

\section{On the trail of Goldstone bosons}

\subsection{The $\sigma$-model}

The Goldstone theorem provides a clear presentation of the physical consequences of SSB on which the Higgs mechanism relies. When the global symmetry of a scalar field is spontaneously broken, the theorem predicts the appearance of a massless spinless particle. ${ }^{3}$ However, a conundrum remains: no such particle has been detected yet and, if it were to exist, the energies at which we would detect it would have already been reached in our apparatuses. This section thereby aims to analyse the problematic physical interpretation of Goldstone bosons. For our purposes, the linear $\sigma$-model at the classical level provides a sufficient, clear introduction to the results about the Goldstone theorem.

Consider the following Lagrangian density, which describes the dynamics of a N-

\footnotetext{
${ }^{1}$ For further references, see Earman (2004a).

${ }^{2}$ The term "heuristic" refers to interacting theories that have not yet been mathematically well defined.

${ }^{3}$ An important distinction exists between global and local symmetries: both are variational, but the former pertain to finite dimensional symmetry groups and the latter to infinite ones. In the Higgs mechanism, the symmetries are local, which is particularly problematic (see Sec. 5.1).
} 
component real scalar field $\Phi=\left(\Phi_{1}, \ldots, \Phi_{N}\right)$ :

$$
\mathcal{L}_{1}=\frac{1}{2} \partial_{\alpha} \Phi . \partial^{\alpha} \Phi+\frac{1}{2} \mu^{2}(\Phi)^{2}-\frac{\lambda}{4}\left((\Phi)^{2}\right)^{2}
$$

where $\mu$ and $\lambda$ are coupling constants, and $\lambda>0$ implies that the Lagrangian is bounded for negative energies. Crucially, this last condition ensures that a vacuum state exists. On the other hand, $\mathcal{L}_{1}$ is invariant under the global $O(N)$ symmetry transformation: $\Phi_{n} \rightarrow R_{n m} \Phi_{m}(n, m=1, \ldots, N)$, with $R^{T} R=I$.

Now, let us focus on the potential $V(\Phi)=-\frac{1}{2} \mu^{2}(\Phi)^{2}+\frac{\lambda}{4}\left((\Phi)^{2}\right)^{2}$, and assume the special case where the mass squared parameter is negative: $\mu^{2}=-m^{2}>0 .{ }^{4}$ The potential may be re-expressed as $V(\Phi)=\frac{\lambda}{4}\left((\Phi)^{2}-v^{2}\right)^{2}$, with $v=\frac{\mu}{\sqrt{\lambda}}$, where we shifted the Lagrangian density by an innocuous constant. The function $V(\Phi)$ reaches its minimal value when:

$$
\Phi_{0}^{2}=v^{2}
$$

Thus we are left with the choice of selecting a particular vacuum state $\Phi_{0}=(0, \ldots, 0, v) .^{5}$ Section 2 emphasized that this choice is arbitrary without the cosmological interpretation since one may take $\mathrm{N}$ different components. However, this choice is sufficient because of the requirement of a unique vacuum state in QFT. At this point, the vacuum state no longer preserves the $O(N)$ symmetry, but the unbroken symmetry group $O(N-1)$ which acts on the first N-1 components of $\Phi_{0} \cdot{ }^{6}$

The physical consequences of SSB arise explicitly by imposing small perturbations $\sigma$ in every direction around $\Phi_{0}$ :

$$
\Phi=\left(g_{n}, v+h\right)=\Phi_{0}+\sigma, \quad \sigma=\left(g_{n}, h\right), \quad n=1, \ldots, N-1
$$

We can rewrite the Lagrangian density in its new form:

$$
\begin{aligned}
\mathcal{L}_{2}= & \frac{1}{2}\left(\partial_{\alpha} g_{n}\right)^{2}+\frac{1}{2}\left(\partial_{\alpha} h\right)^{2} \\
& -\mu^{2} h^{2}-\sqrt{\lambda} \mu h^{3}-\sqrt{\lambda} \mu\left(g_{n}\right)^{2} h-\frac{\lambda}{2}\left(g_{n}\right)^{2} h^{2}-\frac{\lambda}{4} h^{4}-\frac{\lambda}{4}\left(\left(g_{n}\right)^{2}\right)^{2}
\end{aligned}
$$

so that the massive and massless field components become explicit. Only the field $h$ has gained a mass according to the quadratic term $\mu^{2} h^{2}$, while the N-1 massless Goldstone bosons $g_{n}$ are left massless.

It is noteworthy that, in the previous physical derivation, the form of the scalar fields is

\footnotetext{
${ }^{4}$ It is worth pointing that this imaginary mass poses serious difficulties (see Lyre, 2008).

${ }^{5}$ We used two short-cuts here for simplification purposes: 1) we normally deal with vacuum expectation values of fields $\Phi_{0}=\langle 0|\Phi| 0\rangle$ in the quantum case; 2) we assimilate the vacuum state and vacuum expectation value (VEV) of a field since the eigenvalues $\Phi_{0}$ in the vacuum are distinct, and each is respectively associated with one vacuum state.

${ }^{6}$ In the case where $\mu^{2}=-m^{2}<0, \Phi_{0}=0$ and the vacuum state conserves the full $O(N)$ symmetry.
} 
neither significant nor fundamental, since they could even correspond to a pair of fermionic fields. Furthermore, the distribution between massless and massive particles is only a matter of group theory. The Goldstone theorem (Goldstone, 1961) predicts that, if $\mathrm{H}$ is the unbroken subgroup of $\mathrm{G}$, then the number of Goldstone bosons is $\operatorname{dim}(G)-\operatorname{dim}(H)$; that is, there must be one massless scalar field for each spontaneously broken global symmetry. This is a powerful and general result which applies whenever a non-vanishing vacuum expectation value (VEV) arises from a phase transition (see Englert, 2005, 6).

\subsection{Philosophical concerns about the physicality of Goldstone bosons}

Historically, the prediction of massless spinless particles was regarded with trepidation by the physics community. In particular, their existence was deemed impossible due to the fact that electromagnetism and gravitation were the only known long-range interactions with massless mediating fields. Severe doubts were therefore shed on SSB processes and any attempt to observe spontaneously broken symmetries was discouraged. There is indeed something of a paradox, as the presence of apparently physical entities that we have never observed raises the question of whether we are indeed dealing with purely mathematical artefacts.

The temptation is to understand the Goldstone bosons as infinite wavelength oscillations that connect the distinct vacuum states, based on the analogy with the ferromagnet (see Sec. 2). ${ }^{7}$ Because Goldstone bosons are massless, the momentum $k$ tends to zero, so does the frequency, while the wavelength tends to infinity. This means that the propagation requires no energy, a rather peculiar feature which may be illustrated by the instantaneous rotation of an unstable set of spins (see Ruetsche, 2011, 321-322). One may also be tempted to justify this analogy by using the usual unitary transformation of the vacuum state under the action of the conserved charge $Q_{a}$. Each conserved charge is associated with a global broken symmetry with an infinitesimal parameter $\xi_{a}$ (Goldstone et al., 1962, 969):

$$
\left(\exp \left(i \xi_{a} Q_{a}\right)\right)|0\rangle \simeq|0\rangle+i \xi_{a} Q_{a}|0\rangle
$$

The conditions $Q_{a}|0\rangle \neq 0$, associated with each Goldstone mode, entail the existence of many vacuum states. Technicalities aside, this result means that the massless fields manifest indirectly the transition between the various vacua.

However, the previous argument is fallacious, because the charge $Q_{a}$ has an infinite norm on the Hilbert space, which means that the transformation is not unitarily implemented on states. The algebraic formulation, briefly mentioned in Sec. 2, provides formal insights. Goldstone bosons map distinct projections of the algebra of observables on or-

\footnotetext{
${ }^{7}$ An infinite wavelength oscillation is not necessarily an unphysical object, it may correspond to the fact that some underlying medium experiences a uniform phase.
} 
thogonal and therefore disjoint Hilbert spaces, each containing a unique vacuum state. This disjointedness, contrary to analogous finite systems like the ferromagnet, arises from the infinite volume limit and infinite degrees of freedom of relativistic QFT systems (Earman, 2004a). Literally, Goldstone bosons make "steps out of the physical formalism", and should be deemed unphysical in the case of global symmetries. The physical explanation arises from the fact that an infinite energy is required for quantum tunnelling between the distinct vacua. A solution to the issue of purely massless Goldstone bosons will be provided by the Higgs mechanism. ${ }^{8}$

\section{The role of the Higgs mechanism in saving the Goldstone Theorem}

During the early stage of development of gauge theories, physicists realized that massless gauge fields and therefore long range interactions were required by the gauge invariance principle (see Eqs. (6) and (9) below). This contradicted the short-range pattern of the weak force: mediating particles were observed to have a finite lifetime according to the heuristic time-energy uncertainty principle, a fact that required them to be massive. The Higgs mechanism provided a way of avoiding this apparent dead-end. A supplementary scalar field, now called the Higgs field, was introduced to break spontaneously the local gauge symmetry. This led to a short range pattern of the weak force while retaining a gauge invariant Lagrangian. ${ }^{9}$ At the same time, the Higgs mechanism explained why the Goldstone bosons were not being detected, for they were cancelled by choosing a relevant gauge fixing condition. The Higgs mechanism therefore renewed the appeal of SSB.

Given the main motivations of the Higgs mechanism, we derive the case of non-abelian gauge theories with massless gauge fields $A^{\mu}$, expressed in the adjoint representation of the Lie algebra of a group $\mathrm{G}$ as $A^{\mu}=A_{a}^{\mu} t_{a}, a=1, \ldots, \operatorname{dim} G$. Here, the $t_{a}$ are the generators of the Lie algebra. For the Electroweak model (EW), $G=S U(2) \times U(1)$.

For any set of scalar fields $\Phi_{n}$, the local transformation is expressed infinitesimally as

$$
\Phi_{n} \rightarrow\left(1+i \epsilon_{a}(x) t_{a}\right)_{n m} \Phi_{m}
$$

and the gauge Lagrangian

$$
\mathcal{L}_{\text {gauge }}=-\frac{1}{4} F^{\mu \nu} \cdot F_{\mu \nu}, \quad F_{\mu \nu a}=\partial_{\mu} A_{\nu a}-\partial_{\nu} A_{\mu a}+g c_{a b c} A_{\mu b} A_{\nu c}
$$

where $\epsilon_{a}(x)$ are space-time dependent parameters, $g$ is the coupling constant of the gauge fields, and $c_{a b c}$ the structure constants defined by the relation $\left[t_{a}, t_{b}\right]=c_{a b c} t_{c}$.

\footnotetext{
${ }^{8}$ An intuitive solution is also produced by approximate symmetries (Weinberg, 1996).

${ }^{9}$ The idea of the Higgs mechanism was formulated independently at the same time by Englert and Brout (1964), Higgs (1964), and Guralnik et al. (1964).
} 
Scalar fields $\Phi=\left(\Phi_{n}\right)$ are then introduced similarly to the $\sigma$-model (cf. Sec. 3). One purports to use a suitable pattern of SSB, and thereby a suitable non-vanishing VEV, to give mass to the relevant gauge fields (e.g., a massless photon but a massive $Z$ mediating particle). The crucial assumption of local gauge invariance implies that the scalar fields couple to the gauge field $A_{\mu}$ (compare the dynamics with Eq. (1)):

$$
\mathcal{L}_{\Phi}=\frac{1}{2} D^{\mu} \Phi D_{\mu} \Phi-V(\Phi), \quad D_{\mu} \Phi=\partial_{\mu} \Phi+g A_{\mu a} t_{a} \Phi
$$

with gauge transformations given by:

$$
A_{\mu a} \rightarrow A_{\mu a}-\frac{i}{g}\left(D_{\mu} \epsilon\right)_{a}, \quad\left(D_{\mu} \epsilon\right)_{a}=\partial_{\mu} \epsilon_{a}+g c_{a b c} A_{\mu b} \epsilon_{c}
$$

The full theory, $\mathcal{L}_{\Phi}+\mathcal{L}_{\text {gauge }}$, is therefore locally gauge invariant. Note that a mass term $m^{2} \Phi^{2}$ in $\mathcal{L}_{\text {gauge }}$ would not be locally gauge invariant. The issue of mass is solved by assuming a pattern of SSB, with $\mathrm{H}$ the unbroken group of $\mathrm{G}$, such that $V(\Phi)$ has a minimum $\Phi_{0} \neq 0$. The scalar fields are expanded around a chosen vacuum state similarly to the $\sigma$-model:

$$
\Phi=\Phi_{0}+\sigma
$$

So far, the theory still contains non-physical degrees of freedom (redundancies), since the choice of gauge fields is left undecided by the gauge transformations in Eq. (9). The massless scalar fields can be cancelled and the gauge freedom fixed by choosing the unitary gauge:

$$
\left(t_{a} \Phi_{0}\right) . \Phi=0
$$

Roughly speaking, this condition restricts the form of the scalar fields $\Phi$ by linking them to the generators $t_{a}$ of the local symmetries. Since the scalar and the gauge fields are coupled, the restriction on the scalar fields fixes the gauge freedom of the gauge fields.

The unitary gauge offers the advantage of making the physical content of the theory explicit. For this, we decompose the generators and gauge fields using appropriate bases which separate respectively the unbroken group H from the broken group of G:

$$
t_{a}=\left(h_{i}, \hat{t}_{a}\right), \quad A_{\mu a}=\left(A_{\mu i}, \hat{A}_{\mu a}\right)
$$

After making a few computations in the unitary gauge, the covariant derivative reads as

$$
D_{\mu} \Phi=\mathcal{D}_{\mu} \sigma+g \hat{A}_{\mu a} \hat{t}_{a}\left(\Phi_{0}+\sigma\right), \quad \text { with } \quad \mathcal{D}_{\mu} \sigma=\partial_{\mu} \sigma+g A_{\mu i} h_{i} \sigma
$$


and the Lagrangian

$$
\mathcal{L}_{\Phi}=\frac{1}{2} \mathcal{D}^{\mu} \sigma \mathcal{D}_{\mu} \sigma+\frac{1}{2} g^{2} \hat{A}^{\mu a} \hat{A}_{\mu b}\left(\hat{t}_{a}\left(\Phi_{0}+\sigma\right)\right)\left(\hat{t}_{b}\left(\Phi_{0}+\sigma\right)\right)-V\left(\Phi_{0}+\sigma\right)
$$

As a final step, the massive and massless distribution of the theory is given by the quadratic terms of $\mathcal{L}_{\Phi}$ and $\mathcal{L}_{\text {gauge }}$ :

$$
\mathcal{L}_{q u a d}=-\frac{1}{4}\left(F^{\mu \nu} \cdot F_{\mu \nu}\right)_{q u a d}+\frac{1}{2} \partial^{\mu} \sigma \partial_{\mu} \sigma-\frac{1}{2} \sigma \cdot(\mathcal{M} \sigma)+\frac{1}{2} \hat{\mathcal{M}}_{a b} \hat{A}_{a}^{\mu} \hat{A}_{\mu b}
$$

with $\mathcal{M}$ being the mass matrix of the $\sigma$ perturbation (cf. the mass distribution of Sec. 3 ), and $\hat{\mathcal{M}}_{a b}=g^{2}\left(\hat{t}_{a} \Phi_{0}\right)\left(\hat{t}_{b} \Phi_{0}\right)$ the mass matrix of the massive gauge fields.

In the previous derivation, the unitary gauge fixing condition suppresses the Goldstone bosons and gives appropriate masses depending on the initial number of these (the form of $\mathcal{M}$ and $\hat{\mathcal{M}}_{a b}$ is determined by $\mathrm{G}$ and $\mathrm{H}$ and thus by $\hat{t}_{a}$ and $h_{i}$ ). This results in explicit physical contributions, but how arbitrary is that manoeuvre, since nothing prevents the imposition of another gauge fixing condition? The physics should be independent of this choice, which raises several concerns about a realistic interpretation of the gauge bosons "eating" the Goldstone bosons. There are indeed other gauge fixing conditions which leave the Goldstone bosons in the formalism (Sec. 6).

The important point is that Goldstone bosons are not mathematically distinguishable from gauge degrees of freedom: firstly because, as seen previously, they couple to gauge fields, and secondly because they can take the form of gauge degrees of freedom. The Goldstone degrees of freedom can be extracted from the scalar fields as parametrized local transformations acting on the scalar fields (see Weinberg, 1996, 211-225). A redefinition of the parameter in Eq. (6) fully redefines the Goldstone bosons as gauge degrees of freedom, and a suitable gauge fixing condition therefore suppresses them. This calls for comment: the change from global to local symmetries implies that the status of the Goldstone bosons has changed from unphysical entities to redundant degrees of freedom. In other words, the Goldstone degrees of freedom are allowed to change from place to place in the formalism. The consequences of this are crucial: we will see in Sec. 6 that, in perturbation theory, one does not even need to fix the gauge, in order for the Goldstone bosons to give gauge fields their mass.

\section{Gauge matters and theoretical determinations}

The previous exposition has shown that interpreting the Higgs mechanism depends on the relation between the gauge fixing condition and the Goldstone bosons. It is thus important to examine the issue of gauge freedom, and understand with how much arbitrariness and mathematical artefacts the mechanism is strewn. In Sec. 5.2, I will develop and contrast this with the fact that the Higgs mechanism is still seen as the best way of giving the 
gauge fields their mass.

\subsection{Shuffling the variables and breaking the local gauge symmetries}

Considerable doubt has been shed on the possibility of producing a realistic interpretation of the Higgs mechanism, particularly with regard to the significance of the steps presented in Sec. 4, from Eq. (8) to Eq. (15). For instance, $\mathcal{L}_{\text {gauge }}+\mathcal{L}_{\Phi}$ is seen as mathematically the same after being expanded around the vacuum in Eq. (10). This supports the argument that the Higgs mechanism corresponds to "a mere shuffling of degrees of freedom" (Lyre, 2008, 7); that is, a mathematical trick. But one should be cautious about interpreting transformations on degrees of freedom. The fact that the physical degrees of freedom are conserved does not mean that the transformation has no physical grounding. In the present case, the "mere shuffling" view indeed assumes that (i) the choice of a vacuum state, (ii) the redefinition of the fields in terms of small perturbations around the vacuum state, and (iii) the gauge fixing conditions, are not physical. One can, therefore, talk about mathematical transformations up to "a different choice of variables and gauge" (Lyre, 2008, 7), but the argument thus appears circular. As previously seen, the physical counterpart of the choice of a vacuum depends on the cosmological interpretation of the Higgs mechanism (cf. Sec. 2), which is not our current theme here. On the other hand, the previous section demonstrated that the status of the gauge fixing condition is rather ambiguous as well. The issue of gauge symmetries has been widely discussed in the philosophical literature (e.g, Brading and Brown, 2004; Earman, 2004b; Smeenk, 2006), and I will only sketch three main concerns here: 1) the empirical status of local gauge transformations, 2) gauge redundancy, and 3) breaking spontaneously local gauge transformations.

1) First, it is usually assumed in discussions about the Higgs mechanism that the local symmetries like in Eq. (6) have no empirical manifestation contrary to global symmetries. ${ }^{10}$ This feeds into the argument that breaking a "conventional [local] gauge symmetry" (Lyre, 2008, 3) places serious restrictions on a realistic account of the Higgs mechanism. What about the significance of the VEV of the Higgs bosons, and the degeneracy of the vacuum state? Yet, the empirical status of local gauge symmetries is ambiguous, and simply assuming that they are conventional raises many challenges (see Brading and Brown, 2004; Healey, 2007; Greaves and Wallace, 2014). Local gauge symmetries are indeed usually assumed to have at least an indirect physical significance. In the present case, the way in which the symmetry is broken changes the physical content of the theory; namely the number of massless and massive gauge fields.

2) A more serious problem arises from the gauge degrees of freedom (redundancies) proper to the gauge fields (see Eq. (9)). It is widely accepted in the literature that the theoretical descriptions related by local gauge transformations are physically equivalent

\footnotetext{
${ }^{10}$ Note that this is another argument against the analogy with the ferromagnet, cf. Sec. 2.
} 
(Earman, 2004a; t'Hooft, 2007; Struyve, 2011). Earman (2004a,b) emphasized the undetermined physical content of the Higgs mechanism in the absence of an explicit treatment in gauge invariant terms, which Struyve (2011) solved via the constrained hamiltonian formalism. In fact, Higgs (1966) had already provided a gauge independent form of the abelian case without physical loss, and Kibble (1967) extended partially this result to non-abelian theories by isolating the gauge variables corresponding to the degeneracy of the vacuum state.

3) Last but not least, the Higgs mechanism is deemed not to involve a pattern of SSB. Contrary to global symmetries, broken local gauge symmetries leave the vacuum state invariant (Englert, 2005; t'Hooft 2007, 38). The different configurations of the vacuum are thus left indistinguishable. The physical explanation rests on the fact that the Goldstone modes have turned into gauge degrees of freedom (Sec. 4): here, I take the degenerate vacuum state as the existence of independent Goldstone bosons rather than a simple nonvanishing VEV. This simply means that the Higgs mechanism does not involve distinct physical phases, contrary to the Goldstone theorem.

A dilemma is thus offered to us from 3): (A) SSB does not really occur, the Goldstone degrees of freedom are mathematical artefacts of the same type as the gauge redundancies, and the Higgs mechanism is in fact a mathematical trick; or (B) the apparent absence of $\mathrm{SSB}$ is due to the fact that the global symmetries turn into local ones when the scalar and gauge fields couple. Goldstone bosons are really absorbed by the gauge fields, and their convenient formulation as gauge degrees conceals a physical phenomenon that I will address in Sec. 6.

\subsection{Theoretical determinations}

View (A) appears excessive and, before providing arguments for (B) in Sec. 6, I will qualify the view according to which the Higgs mechanism only offers a mathematical, "ad-hoc" trick. For this, let us widen the scope of our analysis slightly by providing a chain of consistent arguments which makes explicit why the Higgs mechanism is seen as the best solution to the mass problem in the Standard Model. We restrict the examples to the case of EW:

(1) There exist quantum numbers that characterize interaction forces (e.g. isospin). They are conserved during the decays between different elements of matter and also specify the types of particles which are sensitive to the interaction (e.g. left-handed leptons and their related neutrinos): Positive argument.

(2) The values of these quantum numbers are symmetrically related, so one may study them as n-uplets representations of a symmetry group (e.g., on the basis of the isospin quantum number, the left-handed electron and its neutrino regrouped in a doublet form a two dimensional representation of $S U(2))$ : Theoretical convenience. 
(3) The theory should be invariant under the symmetry transformations between different n-uplets; that is, the theory describes all of the possible ways in which particles of a same family can interact (e.g., the Lagrangian governing the $\beta$-decay $p \rightarrow n$ should be invariant under $S U(2)$, and the lepton number is conserved through the decay $\mu^{-} \rightarrow e^{-}+\bar{\nu}_{e}+\nu_{\mu}$ ): Theoretical convenience (unified description of similar particles).

(4) The only known way to describe interactions (relations between symmetric n-uplets) is achieved by postulating local gauge transformations and a gauge invariant Lagrangian that couple gauge fields and matter fields (cf. Sec. 4). On the contrary, global transformations would only change the phase convention of the matter fields: Nomological sufficiency. ${ }^{11}$

(5) Local gauge symmetries and gauge invariance require that mediating particles are massless: Nomological necessity.

(6) Experiments show that some fundamental interactions are short-ranged (e.g, the weak interaction), a pattern which corresponds to a finite lifetime of mediating particles, as explained in Sec. 4. This result is best accommodated by postulating the existence of massive mediating particles so that their propagation "slows down" and their decay becomes observable: Positive argument.

(7) Some of the local gauge symmetries of the theory should be broken to allow massive representations to appear in the Lagrangian formulation (e.g., in $\mathrm{EW}, S U(2) \times U(1)$ is broken to $U(1)$ to keep the photons massless and give mass to the $Z$ and $W$ particles): Nomological necessity.

(8) However, adding the usual massive terms $m^{2} A^{\mu} A_{\mu}$ does not preserve the gauge invariance of the theory. The Higgs mechanism circumvents this issue by postulating the effect of external fields whose VEV break spontaneously the local gauge symmetries: Nomological sufficiency.

Although such a chain is debatable, what becomes clear is the weakness of the nomologically sufficient arguments for interpreting the Higgs mechanism realistically: namely, the hypothesis of the local gauge transformations, gauge invariance (4), and introduction of external fields (8). These arguments are weak, as they introduce explicitly theoretical underdetermination; that is, there might be several but equivalent formulations of the same theoretical matter.

On the one hand, the justification of the gauge principle has raised important concerns (Kosso, 2000; Martin, 2003), since it appears as the main rationale for the Higgs

\footnotetext{
${ }^{11} \mathrm{By}$ 'nomological sufficiency', I mean theoretical arguments that are sufficient but not necessary to give a consistent answer to a law-like problem.
} 
mechanism to hold. The Goldstone theorem had already introduced an inconvenient gap between particle phenomenology and theory, but the Higgs mechanism increases the problem even further by cancelling out unphysical particles. Does the Higgs mechanism save a theoretical concept rather than possible phenomena (Kosso, 2000, 367)? The gauge principle is fundamental though, as the only known renormalizable and working theories are locally gauge invariant. On the other hand, concerning (8), dynamical symmetry breaking (DSB) is a more intrinsic and less ad-hoc way to break spontaneously a symmetry: this mechanism does not require an additional structure like the scalar fields of the Higgs mechanism. However, DSB introduces other complications (see Weinberg, 1996), and recent experimental detections favour elementary scalar fields, even though their form has not yet been experimentally determined.

All of these previous concerns, allied to the gauge issues of subsection 5.1, may have seemed to cast doubts on the Higgs mechanism. Nonetheless, this chain of consistent arguments underscores the logical motivations, yet schematic here, which give credit to the Higgs mechanism, not to mention that it also proved an efficient tool for the unification of the fundamental forces (Peskin and Schroeder, 1995). We will now examine (B).

\section{Examining the heuristics}

The present section claims that the gauge issues do not undermine the metaphor that the Goldstone bosons are "eaten" by gauge fields. For a continuous class of gauge fixing conditions, the Goldstone bosons are cancelled in perturbation theory before the gauge is fixed, so that the gauge fixing condition appears to be a short-cut. The Goldstone Bosons Equivalence theorem completes the argument by contesting that the cancellations are merely unphysical. Hereafter, my strategy is to follow the illustrations about the Higgs mechanism as an Ariadne's thread to move away from the intricacies of gauge theories and specify the underlying physical phenomenon.

The philosophical literature has too quickly derided these illustrations in its search for conceptual clarification (hereafter referred to as the "just-so stories", Earman, 2004b, 1239). Amongst others lies the previous suspicious metaphor that gauge fields "eat" the Goldstone bosons and thereby become massive when the symmetry is broken, but nothing in the previous derivation of Sec. 4 supports this metaphor: the trick is rather to fix "by hand" a suitable gauge which suppresses the Goldstone bosons. The metaphor is thus clearly too sensational, but yet too easily derided. One should rather question on which physical intuitions these illustrations rely, and examine how they substantiate the formal derivation.

Prior to that, let us make explicit the moral of the aforementioned just-so story. It tells us that the concept of mass no longer needs to be added "by hand" to the theory: we are 
shifting towards a view whereby the concept of mass is extrinsic rather than intrinsic. ${ }^{12}$ Surely there is no final decision about the truth of this moral. In particular, the numerical values of the different masses of matter fields are not fixed by the Higgs mechanism, since it leaves the Standard Model with the same number of free parameters (Lyre, 2008). However, in epistemological terms, the Higgs mechanism seems to increase the explanatory power of particle physics, at least by challenging the received orthodoxy that mass is intrinsic.

The moral of the just-so story has the advantage of making particularly salient the physical intuition underlying the Higgs mechanism: a third part must give particles their mass property. In high energy physics, an apparent mass arises when the movement of particles is refrained. Here, two additional illustrations might elucidate the nature of the link in question: particles are pictured as acquiring mass thanks to a spoon dipping in the Higgs marmalade or to the difficulty of crossing a crowded cabinet (Lyre, 2012, 4). I take these two stories to underline two different patterns. (i) The third part, the marmalade, interacts directly with the particles and refrains them. At high energies, this pattern is well-illustrated by radiative corrections, where energy fluctuations, usually depicted as a cloud of virtual particles, interact continuously with particles and affect their parameters. (ii) The interaction that drives particles is screened by an indirect background effect (assuming that the crowd simply blocks the way through the cabinet). A canonical example of a screening effect is, for instance, when the electromagnetic field produced by a particle is damped by the field produced by other surrounding charged particles.

From the two patterns (i) and (ii), it is possible to propose relationist and substantialist perspectives of the Higgs mechanism:

(i) From a relationist perspective, the Higgs bosons are continuously interacting with gauge particles, as illustrated by the terms $\frac{1}{2} g^{2} \hat{A}^{\mu a} \hat{A}_{\mu b}\left(\hat{t}_{a}\left(\Phi_{0}+\sigma\right)\right)\left(\hat{t}_{b}\left(\Phi_{0}+\sigma\right)\right)$ in the Lagrangian $\mathcal{L}_{\Phi}$ in Eq. (14). One may expect energy fluctuations of the Higgs field in its vacuum state, and terms like $\hat{A}^{\mu a} \hat{A}_{\mu b} \sigma^{2}$ allow us to picture the propagation of gauge fields as laced with Higgs bosons. From the previous account, the physical role of Goldstone bosons and the conceptual challenges of the non-vanishing VEV of the Higgs field remain unclear, and I will briefly clarify them later in this section. On the experimental side, when particles collide with sufficient energy, a Higgs boson may be released and freely decay in a very short time, so that we may observe it.

(ii) The substantialist view relies on the significance of the non-vanishing VEV and on the analogy with vacuum bubbles. The vacuum is usually understood as containing fluctuating polarization processes with the creation of particle-antiparticle pairs, which arise either inside interactions or outside as independent bubbles (cf. the $\Phi^{4}$

\footnotetext{
${ }^{12}$ By 'intrinsic', I mean that the mass is the 'own' property of a particle, and not dependent on the environment.
} 
self-interaction terms in Eq. (1), Sec. 3). The issue with the Higgs mechanism is that, even when the Higgs fields are not excited, their non-vanishing VEV give a mass to the gauge fields. This does not really correspond to a dynamical coupling, since the VEV is an average of the fluctuations in the vacuum and appears as a constant in the Lagrangian. One may speculate that the gauge fields are "screened" by a Higgs background effect. The fundamental aspect of this view is that this background, composed of interacting "bubbles", could be directly probed when the energy of collisions becomes sufficient. Unfortunately, because external incoming particles are required to collide, one may be unable to distinguish whether the vacuum bubbles are independent of the interaction or not, and thus decide between (i) and (ii) from experiments.

So far, the previous account shows how stories provide interesting physical intuitions and substantiate some of the couplings in the Higgs mechanism. However, the effect of Goldstone bosons does not seem to be included and the non-vanishing VEV are left unexplained, though it distinguishes between the substantialist and relationist views. For this, let us specify now the first of these two concerns. The second is highly complex, and I will only provide brief insights into it at the end.

Before the unitary gauge is fixed, the absorption and emission of Goldstone bosons can be shown to have a formal counterpart. They are annihilated and created by the conserved currents of the broken symmetries. When these currents couple to gauge bosons, the latter become massive. I take these relations as a hint that the Goldstone bosons surely play a physical role in the Higgs mechanism.

Consider the conserved current $\mathcal{J}^{\mu a}(x)$ of the broken symmetry to which a Goldstone boson is coupled with strength $F_{n}^{a} .\left|G_{n}\right\rangle$ is the $n^{\text {th }}$ Goldstone boson state and $p$ its momentum. This current can be viewed as annihilating or creating the boson (for more details, see Peskin and Schroeder, 1995, chap. 20; Englert, 2005):

$$
\left\langle 0\left|\mathcal{J}^{\mu a}(x)\right| G_{n}(p)\right\rangle=-i p^{\mu} F_{n}^{a} e^{i p . x}
$$

Now, the first order interaction terms between the currents and the gauge fields $A_{\mu}^{a}$ can be written as $g A_{\mu}^{a} \mathcal{J}^{\mu a}$, so that the probability amplitude for the $n^{\text {th }}$ Goldstone boson to be "absorbed" by a gauge boson of momentum $k$ is $g k^{\mu} F_{n}^{a}$. Therefore, there is a massless pole contribution to the self-energy of the gauge fields (Fig. 1):

$$
\left(+g k^{\mu} F_{n}^{a}\right) \frac{i}{k^{2}}\left(-g k^{\nu} F_{n}^{b}\right)
$$




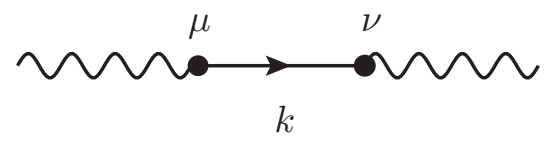

Figure 1: The Feynman diagram of the gauge fields (wave lines) absorbing the Goldstone bosons (straight lines).

On the other hand, a similar term arises from the Lagrangian in Eq. (8) allied with Eq. (10) before the gauge is fixed. Interactions between Goldstone bosons and gauge fields of the form $g A_{a}^{\mu}\left(\hat{t}_{a} \Phi_{0}\right) . \partial_{\mu} \sigma$ give a leading order contribution to the self-energy of the gauge fields:

$$
\left(+g k^{\mu}\left(\hat{t}_{a} \Phi_{0}\right)_{n}\right) \frac{i}{k^{2}}\left(-g k^{\nu}\left(\hat{t}_{b} \Phi_{0}\right)_{n}\right)
$$

Or $\hat{\mathcal{M}}_{a b}=g^{2}\left(\hat{t}_{a} \Phi_{0}\right)\left(\hat{t}_{b} \Phi_{0}\right)$ is the gauge field mass matrix derived in Eq. (15), Sec. 4. Identifying the terms in Eq. (17) and Eq. (18) gives:

$$
g^{2} F_{n}^{a} F_{n}^{b}=\hat{\mathcal{M}}_{a b}
$$

The previous derivation shows two things: (i) Goldstone bosons contribute to the dynamics of gauge fields; (ii) the capacity of Goldstone bosons to be absorbed and annihilated gives back the expression of the mass of gauge fields. It is difficult to develop this interpretation further though. It is as if the Goldstone bosons were "carrying" mass from the Higgs vacuum to the gauge fields. There is a more technical way of formulating this point: the Goldstone bosons provide a longitudinal polarization for the gauge fields, ${ }^{13}$ which physically means that they become real massive fields. Yet, the theory still contains unphysical degrees of freedom since the gauge has not been fixed.

The Faddeev-Popov method for deriving a quantized gauge field theory from the path integral approach provides further insights into this issue. In this method, the gauge fixing condition does not need to be fixed immediately, though its form is restricted to satisfy a renormalizable theory. A gauge parameter $\xi$ can be kept throughout, so that a continuous class of gauges is left for us to choose, amongst them the unitary gauge (Peskin and Schroeder, 1995, chap. 21; Weinberg, 1996, chap. 15). Of course, some fundamental properties are lost: for instance, the introduction of non-gauge-invariant terms and a certain number of unphysical contributions as auxiliary fields, but the description is kept at a very general level. The derivation for spontaneously broken gauge field theories,

\footnotetext{
${ }^{13}$ Massive vector fields have four polarization states: one time-like, two transverse and one longitudinal.
} 
rather technical in nature, produces the following result: the unphysical contributions of the gauge fields exactly cancel those of the Goldstone bosons at any order of perturbation theory. More precisely, the Goldstone bosons cancel the unphysical time-like polarization of the gauge fields, and leave the transverse and longitudinal polarizations untouched. Since the time-like and longitudinal polarization cancel each other out in massless gauge theories, one may therefore say that, in massive gauge theories, the Goldstone bosons prevent the gauge fields to become massless.

Here comes the benefit of not having fixed the gauge parameter $\xi$. The different gauge fixing conditions should be interpreted as perspectives rather than arbitrary choices, for the theory satisfies different fundamental assumptions of QFT (e.g., Lorentz invariance, unitarity) in different gauges, and only the whole class of gauges leads the theory to be consistent. In addition, each gauge provides a different perspective on the relation between the Goldstone bosons and the longitudinal polarization of the gauge fields, though the underlying physics remains unchanged. In particular, the unitary gauge $(\xi \rightarrow \infty)$ shows the physical outcome that one expects: namely, that the Goldstone bosons vanish and only the physical polarizations of the gauge fields are present. More interestingly, the Lorentz gauge $(\xi=0)$ shows that, all other things being equal, the longitudinal polarization is replaced by a massive Goldstone boson. Should we interpret this as an interchange between this polarization and a Goldstone boson? So far, our account displays rather cancellations of unphysical degrees of freedom than proper interactions between physical fields. The same decay amplitudes are indeed obtained with the different gauges, so there is no empirical ground for the Goldstone bosons yet.

The fundamental result which reinforces the view that Goldstone bosons are physically absorbed is given by the Goldstone Boson Equivalence theorem (Peskin and Schroeder, 1995, chap. 21.2; Horejsi, 1997). At relativistic speeds, the longitudinal polarization becomes distinct from the transverse polarizations. In this case, the theorem states that "the amplitude for emission or absorption of a longitudinally polarized massive gauge boson becomes equal to the amplitude for emission or absorption of the Goldstone boson that was eaten by the gauge boson" (Peskin and Schroeder, 1995, 744). A straightforward consequence of this is that the longitudinal polarization of the gauge fields has the same coupling structure as that of the Goldstone bosons. This does not necessarily mean that Goldstone bosons may be observed - they are still "would-be" bosons, but it shows that the longitudinal gauge bosons and the Goldstone bosons are interchangeable at high energies. As Horejsi (1997, 3) puts it, Goldstone bosons and longitudinal gauge bosons are "natural partners", in a sense. Their relation is not governed by our choice to apply a certain gauge fixing condition, but appears physical in the sense of having the same emission and absorption patterns. The empirical aspect of the Goldstone bosons requires further study but, for now, the Equivalence theorem seems to support the view that the Goldstone bosons are absorbed by the gauge fields. 
Before concluding, let us briefly mention how the two previous substantialist and relationist accounts may include the idea of Goldstone bosons and VEV of the Higgs fields. In perturbation theory, $\Phi_{0}=\langle 0|\Phi| 0\rangle \neq 0$ corresponds to a "tadpole" Feynman diagram; that is, a one-loop diagram with one external leg. The mass of the gauge fields is corrected by vacuum fluctuations at any energy order and, amongst the many diagrams which represent these corrections, arise some of these tadpoles, connected to the propagation of the gauge fields. It seems, therefore, that the Higgs fields work as a sort of ether, pictured by the vacuum bubbles of tadpoles. On the other hand, the Goldstone bosons seem to be a more relational attribute (or "carriers") of the mass of the gauge fields (cf. Fig. 1 above). In regard to these two remarks, the substantialist and relationist perspectives seem to reconcile. Yet, numerous issues underlie the previous discussion, the vacuum fluctuations, and these diagrams; notably, the question of how the perturbative expansion and virtual particles should be considered in QFT.

\section{Conclusion}

From the above analysis, the following conclusion emerges. What seems dubious in the Higgs mechanism, that the Goldstone bosons are absorbed by the gauge fields to gain mass, has to be reconsidered in the light of the two following points. (i) The cancellations of the time-like polarization of the gauge fields and Goldstone bosons at any order of perturbative expansion contest the importance of the gauge fixing condition in the interpretation of the Higgs mechanism. (ii) The correspondence between the longitudinal polarization of the gauge fields and Goldstone bosons, both by the different gauges in the Faddeev-Popov method and by the Goldstone Boson Equivalence theorem, challenges the view that the absorption is merely unphysical. These two points thus leave opened the question of whether the metaphor is inadequate, and have allowed us to expound several aspects of the metaphor which have been overlooked by the literature. However, further study needs to be done to understand the role of the perturbative expansion and virtual processes in the interpretation of the Higgs mechanism.

\section{Acknowledgements}

I am particularly grateful to Nazim Bouatta and Jeremy Butterfield for detailed comments and helpful discussions on the present essay. I would like to thank Koray Karaca and anonymous referees for their helpful advice and comments.

\section{References}

Baker, D. J. and H. Halvorson (2013). How is spontaneous symmetry breaking possible? Understanding Wigner's theorem in light of unitary inequivalence. Studies in His- 
tory and Philosophy of Science Part B: Studies in History and Philosophy of Modern Physics 44(4), 464-469.

Brading, K. and H. R. Brown (2004). Are Gauge symmetry transformations observable? British Journal for the Philosophy of Science 55(4), 645-665.

Brading, K. and E. Castellani (2003). Symmetries in physics: Philosophical reflections. Cambridge University Press.

Coleman, S. (1985). Aspects of symmetry: Selected Erice lectures of Sidney Coleman. Cambridge University Press.

Collaboration, A. (2012). Observation of a new particle in the search for the Standard Model Higgs boson with the ATLAS detector at the LHC. Physics Letters B 716(1), $1-29$.

Earman, J. (2003). Rough guide to spontaneous symmetry breaking. In K. Brading and E. Castellani (Eds.), Symmetries in physics: Philosophical reflections, pp. 335-346. Cambridge University Press.

Earman, J. (2004a). Curie's principle and spontaneous symmetry breaking. International Studies in the Philosophy of Science 18(2-3), 173-198.

Earman, J. (2004b). Laws, symmetry, and symmetry breaking: Invariance, conservation principles, and objectivity. Philosophy of Science 71(5), 1227-1241.

Englert, F. (2005). Broken symmetry and Yang-Mills theory. In G. t'Hooft (Ed.), 50 Years of Yang-Mills Theory, pp. 65-95. World Scientific Publishing Company.

Englert, F. and R. Brout (1964). Broken symmetry and the mass of gauge vector mesons. Physical Review Letters 13(9), 321-323.

Goldstone, J. (1961). Field theories with superconductor solutions. Il Nuovo Cimento (1955-1965) 19(1), 154-164.

Goldstone, J., A. Salam, and S. Weinberg (1962). Broken symmetries. Physical Review $127(3), 965$.

Greaves, H. and D. Wallace (2014). Empirical consequences of symmetries. British Journal for the Philosophy of Science 65(1), 59-89.

Guralnik, G. S., C. R. Hagen, and T. W. B. Kibble (1964). Global conservation laws and massless particles. Physical Review Letters 13(20), 585-587.

Healey, R. (2007). Gauging what's real: The conceptual foundations of contemporary gauge theories. Oxford University Press. 
Higgs, P. (1966). Spontaneous symmetry breakdown without massless bosons. Physical Review 145(4), 1156-1163.

Higgs, P. W. (1964). Broken symmetries and the masses of gauge bosons. Physical Review Letters 13(16), 508-509.

Horejsi, J. (1997). Electroweak interactions and high-energy limit. Czechoslovak Journal of Physics 47(10), 951-977.

Kibble, T. W. B. (1967). Symmetry breaking in non-abelian gauge theories. Physical Review 155(5), 1554-1561.

Kosso, P. (2000). The Epistemology of spontaneously broken symmetries. Synthese 122(3), 359-376.

Lyre, H. (2008). Does the Higgs mechanism exist? International Studies in the Philosophy of Science 22(2), 119-133.

Lyre, H. (2012). The Just-so Higgs story: A response to Adrian Wüthrich. Journal for General Philosophy of Science 43(2), 289-294.

Martin, C. (2003). On Continuous symmetries and the foundations of modern physics. In K. Brading and E. Castellani (Eds.), Symmetries in physics: Philosophical reflections, pp. 29-60. Cambridge University Press.

Morrison, M. (2003). Spontaneous symmetry breaking: theoretical arguments and philosophical problems. In K. Brading and E. Castellani (Eds.), Symmetries in physics: Philosophical reflections, pp. 347-363. Cambridge University Press.

Peskin, M. E. and D. V. Schroeder (1995). An Introduction to quantum field theory, Volume 94. Westview Press.

Redhead, M. (2003). The Interpretation of gauge symmetry. In K. Brading and E. Castellani (Eds.), Symmetries in physics: Philosophical reflections, pp. 124-139. Cambridge University Press.

Ruetsche, L. (2011). Interpreting quantum theories: The art of the possible. Oxford University Press.

Smeenk, C. (2006). The Elusive Higgs mechanism. Philosophy of Science 73 (5), 487-499.

Struyve, W. (2011). Gauge invariant accounts of the Higgs mechanism. Studies in History and Philosophy of Science Part B 42(4), 226-236.

t'Hooft, G. (2007). The Conceptual basis of quantum field theory. In J. Butterfield and J. Earman (Eds.), Philosophy of Physics, Part A, Handbook of the Philosophy of Science, pp. 661-729. Elsevier. 
Weinberg, S. (1996). The Quantum theory of fields, Vol. 2: Modern applications (1 ed.). Cambridge University Press. 